
internationales

vol. $19-n^{\circ} 3 \mid 2003$

Moyen-Orient : mutations récentes d'un carrefour migratoire

\title{
Un champ à explorer : le rôle des pèlerinages dans les mobilités nationales, régionales et internationales du Moyen-Orient
}

\section{Sylvia Chiffoleau}

\section{(2) OpenEdition}

Édition électronique

URL : https://journals.openedition.org/remi/2695

DOI : 10.4000/remi.2695

ISSN : $1777-5418$

Éditeur

Université de Poitiers

Édition imprimée

Date de publication : 12 décembre 2003

Pagination : 285-289

ISBN : 2-911627-35-0

ISSN : 0765-0752

\section{Référence électronique}

Sylvia Chiffoleau, « Un champ à explorer : le rôle des pèlerinages dans les mobilités nationales,

régionales et internationales du Moyen-Orient », Revue européenne des migrations internationales [En ligne], vol. 19 - n³ | 2003, mis en ligne le 09 juin 2006, consulté le 14 avril 2022. URL : http://

journals.openedition.org/remi/2695; DOI : https://doi.org/10.4000/remi.2695

Ce document a été généré automatiquement le 14 avril 2022.

(c) Université de Poitiers 


\title{
Un champ à explorer : le rôle des pèlerinages dans les mobilités nationales, régionales et internationales du Moyen-Orient
}

\author{
Sylvia Chiffoleau
}

1 Polarisé par les villes saintes de Jérusalem, La Mecque et Médine, l'espace procheoriental est en outre ponctué d'une multitude de lieux sacrés, mosquées et églises prestigieuses ou modestes tombeaux de saints, qui dessinent une dense topographie sacrée, avec ses itinéraires et ses haltes, ses lieux de rencontre ou de croisement, et ses sanctuaires au long et au bout de la route. Les pèlerinages vers ces lieux saints constituent encore aujourd'hui l'une des principales causes de mobilité dans la région. Il s'agit certes de mobilités d'une nature particulière : elles reposent toujours sur un choix délibéré, sont guidées avant tout par une démarche de foi, même si elles s'accompagnent aussi d'autres motivations (commerce, sociabilité familiale ou confessionnelle...) et elles demeurent le plus souvent temporaires.

2 La pérégrination est un trait fondamental de la civilisation arabo-musulmane, laquelle s'est construite dans les frontières d'un empire. Commerce, savoir et religion sont indissociablement liés dès le Moyen-Âge pour engendrer d'importants flux de circulation dont le pèlerinage à La Mecque, l'une des cinq obligations de l'islam, constitue la destination phare. Le caractère obligatoire du hajj, l'absence d'images et de reliques qui place le sanctuaire ou le tombeau au centre de la dévotion, tout cela contribue à créer en islam une véritable « dictature du lieu » (Mayeur-Jaouen, 2000). Ce caractère obligatoire est modéré par la nécessité de pouvoir faire face au voyage, ce qui permet l'existence de sites de substitution, rôle que jouent fréquemment les tombeaux des saints ou des "gens de la famille». Le pèlerinage s'impose pour toute rencontre avec un saint ou un prophète, pour toute intercession ou imprégnation par la baraka émanant des lieux sacrés. La structure du sacré en islam impose le déplacement. 
3 En raison même de leur ampleur, les mouvements liés aux pèlerinages sont étroitement dépendants des questions géopolitiques qui peuvent profondément affecter ou transformer, temporairement ou définitivement, la géographie des lieux saints. La disparition de l'Empire ottoman à la fin de la Première Guerre mondiale a modifié le destin de nombreux pèlerinages : jusqu'alors fréquentés par des populations venues de tout l'empire, leur rayonnement s'est replié dans le cadre des frontières nationales. La création d'Israël et la persistance du conflit israélo-arabe ont limité l'accès de Jérusalem aux Arabes, chrétiens et musulmans. Le contrôle exercé par le pouvoir de Saddam Hussein sur les villes et le culte de Nadjaf et Kerbala, en Irak, a induit un redéploiement de la ferveur chiite envers les membres de la famille du prophète hors des villes saintes traditionnelles. En revanche, depuis une quinzaine d'années, on assiste à un phénomène général de réouverture, à une augmentation et une diversification sensibles des flux pèlerins. La disparition de l'Union soviétique a réintégré l'Asie centrale et le Caucase dans le mouvement des pèlerinages musulmans ; dans les Balkans, la relance ou l'émergence de certains pèlerinages témoigne des réajustements récents opérés entre le religieux et le politique (Clayer et Popovic, 1995). Les effets de la mondialisation se font évidemment sentir sur les mouvements liés aux pèlerinages. D'une part, l'intensification des flux et la baisse du coût des transports offrent des opportunités de mobilité inédites jusqu'alors, bien qu'elles tendent par ailleurs à gommer la notion d'effort de la démarche pèlerine, laquelle se trouve de plus en plus associée à l'idée de tourisme. D'autre part, les mobilités motivées par les pèlerinages donnent prétexte à la mise en place de formes plus ou moins formelles et légales de commerce international. Les changements globaux, qu'ils soient d'ordre politique ou économique, affectent donc de façon sensible les flux et les conditions de déplacement des pèlerins. Ces changements se manifestent à différentes échelles, locale, régionale et internationale, l'ensemble constituant un champ de recherche qu'il conviendrait d'explorer, et que quelques travaux récents ont commencé de baliser.

4 Au niveau local, le culte des saints continue de susciter d'importants déplacements intranationaux. Tout sanctuaire donne lieu à des visites individuelles (ziyâra) et dans certains pays, notamment en Égypte et au Maghreb, des pèlerinages collectifs ont lieu à l'occasion d'événements comme l'anniversaire de la naissance du prophète (mawlid) ou du saint (mawsim), ce qui en revanche n'a pas (ou peu) cours au Levant. La sacralisation temporaire d'un lieu entraîne une série de requalifications de celui-ci. Ainsi, à l'occasion d'un pèlerinage, l'espace urbain se trouve investi par les ruraux ou le monde tribal, qui y recréent leur mode de vie (tentes, sociabilité de proximité...), ou à l'inverse, le monde urbain sort des limites de la ville pour s'installer et reproduire temporairement la vie urbaine dans des espaces ruraux. C'est par exemple le cas à Shihr, dans le Hadramaout, où l'on repère ce type d'investissement du monde tribal par les citadins (Camelin, 2001). Si les pèlerinages locaux manifestent encore une certaine vitalité, ils sont cependant peu à peu érodés par le processus général de sécularisation, par un contrôle politique de plus en plus étroit, par le reflux du soufisme et le relâchement des identités locales. Aussi, le sens même de ces manifestations tend-il à changer. La comparaison entre l'Égypte et le Maghreb serait à ce titre intéressante à pousser. En Égypte en effet, les pèlerinages locaux ne semblent pas (encore ?) trouver de formule efficace de renouvellement. Au Maghreb au contraire, certains d'entre eux sont énergiquement pris en main par le pouvoir politique qui cherche à les folkloriser à l'intention du tourisme international, mais aussi de la population émigrée. Ainsi, on n'hésite pas à modifier la date de tel mawsim pour le placer au cœur de la période 
estivale, au moment où les émigrés sont de retour au pays. C'est ce processus de folklorisation et l'association de plus en plus étroite des pèlerinages au monde du tourisme qui expliquent également le succès des mawsim-s de bord de mer au Maroc (Berriane, 1992).

$5 \mathrm{Au}$ niveau régional, l'effondrement de la barrière idéologique qui coupait les populations musulmanes de l'ex-URSS du bassin naturel d'expression de leur foi a permis de les réintégrer dans les circulations liées à la religion, notamment au pèlerinage à La Mecque dont elles étaient jusqu'alors quasi exclues. La réactivation de réseaux parfois fort anciens à l'occasion des mobilités pèlerines, qui donne lieu à des processus de ressourcement identitaire (Balci, à paraître), mais aussi à la reprise d'échanges commerciaux traditionnels entre l'Asie centrale et le Moyen-Orient, mérite d'être finement étudiée. Le développement récent du site du mausolée de Sa'd al-dîn alJibawî, en Syrie, témoigne du regain de vitalité de certains lieux sacrés, rendu possible en l'occurrence par la liberté de circulation retrouvée par les populations des Balkans, où la voie soufie dont al-Jibawî est le fondateur est fortement implantée (Geoffroy, 1995). La géographie mouvante des pèlerinages chiites est à ce titre particulièrement exemplaire. L'interruption des pèlerinages iraniens vers les villes saintes de Nadjaf et Kerbala en Irak depuis la guerre qui a opposé les deux pays au cours des années quatrevingt, et les obstacles dressés par le pouvoir de Saddam Hussein à la pratique du culte des chiites, ont infléchi les itinéraires pèlerins de ceux-ci en direction de la Syrie. Plusieurs mausolées de ce pays, supposés abriter la dépouille de membres de la famille du prophète, ont été rénovés, voire reconstruits, grâce à des fonds iraniens. Ainsi du mausolée de sayyida Ruqayya, dans la vieille ville de Damas, et de sayyida Zaynab, dans la banlieue de la capitale syrienne, qui draine chaque année plus d'un million de pèlerins (Mervin, 1996). Ces flux toursitico-religieux ont un impact non négligeable sur l'économie du pays. De nombreux hôtels se consacrent exclusivement à l'accueil de ces visiteurs, iraniens pour la plupart, qui accompagnent bien souvent leur périple religieux d'activités commerciales (Adelkha, 1997). Mais cette nouvelle géographie sacrée du chiisme, qui privilégie pour le moment la Syrie, est sans doute à son tour susceptible d'être modifiée par la réouverture des villes saintes d'Irak.

6 Le cas des pèlerinages chiites permet d'évoquer une autre particularité des mobilités religieuses. La plupart des lieux de pèlerinage chiites abritent des écoles religieuses (hawza-s) formant un réseau qui joue un rôle semble-t-il majeur dans la circulation du savoir (Mervin, 1996). Ces centres accueillent des étudiants venant de toutes les parties du monde chiite, créant ainsi des carrefours de rencontres et d'échanges, et certains étudiants suivent des itinéraires de formation dans l'ensemble de la région, attirés par le charisme de tel ou tel clerc, système qui n'est pas sans évoquer les pérégrinations des savants du Moyen-Age. Si le réseau d'écoles du sunnisme ne revêt peut-être pas la même importance, certains lieux de pèlerinage abritent néanmoins des écoles religieuses (c'est le cas par exemple de la ville de Tanta, en Égypte). À l'échelle régionale, d'autres pistes mériteraient bien sûr d'être suivies.

7 Si on insiste ici sur les pèlerinages musulmans, ceux des chrétiens n'en ont pas moins leur importance, suscitant d'ailleurs parfois des rencontres interconfessionnelles, notamment autour des figures de la Vierge et de Saint-Georges. Certains pèlerinages chrétiens permettent d'entretenir les réseaux familiaux et l'identité confessionnelle par-delà les frontières, notamment entre le Liban et la Syrie. D'autres peuvent témoigner de l'influence des migrations sur l'évolution des rituels, puisque certains 
d'entre eux sont aujourd'hui largement fréquentés par une population immigrée venue d'Asie du Sud-Est.

8 Le pèlerinage à La Mecque est bien sûr la principale cause des migrations religieuses à l'échelle internationale. Les flux vers les Lieux saints de l'Islam n'ont cessé d'augmenter au cours du dernier quart du vingtième siècle, en raison de l'amélioration des moyens de transport et de la baisse de leur coût, mais aussi en raison d'une augmentation générale de la pratique religieuse. Depuis le début des années quatre-vingt dix, le groupe des pèlerins s'est enrichi de la présence des musulmans issus des anciennes républiques soviétiques, et ils sont également de plus en plus nombreux à venir depuis les pays d'immigration, l'Europe et les Etats-Unis notamment. Depuis 1987 cependant, l'Arabie Saoudite fixe des quotas pour chaque pays, pourtant le chiffre total des pèlerins qui se rassemblent aujourd'hui à La Mecque dépasse les deux millions d'individus. Le visa de hajj est strictement temporaire, mais le pèlerinage suscite sans doute encore aujourd'hui, comme ce fut le cas tout au long des siècles passés, des installations définitives, dans les villes saintes elles-mêmes, mais aussi au long de l'itinéraire suivi par les pèlerins. En outre, le pèlerinage alimente une part de l'immigration illégale en Arabie Saoudite, notamment parmi la population d'Asie du Sud-est (Husson, 1996). Si le pèlerinage est avant tout une démarche de foi, il s'accompagne parfois d'autres types de motivation et donne lieu à de multiples échanges, commerciaux ou idéologiques. Les grandes foires qui accompagnaient autrefois le pèlerinage ne sont plus de mise, mais celui-ci suscite toujours d'importants flux de commerce international. C'est notamment le cas en Afrique, où le Tchad par exemple a vu naître la figure du pèlerin-commerçant, multipliant les voyages sacrés vers l'Arabie Saoudite d'où sont ensuite importés de façon informelle de multiples biens de consommation, avec la complicité des autorités tchadiennes. Par ailleurs, dans le contexte du pèlerinage, les croyants sont à la fois absorbés dans l'identité collective de la oumma, symbolisée par le port du même vêtement blanc de l'ihrâm, et confirmés dans leur identité nationale en raison de l'organisation même du pèlerinage où tout (transport, logement, encadrement par des guides...) fonctionne par groupes relevant d'un même pays. Cette dualité suscite à la fois la circulation générale d'idées politicoreligieuses, contribuant notamment à la diffusion du modèle wahhabite, et des réactivations identitaires par nationalité. Malgré tout, le pèlerinage à La Mecque ne semble pas être un lieu du politique particulièrement actif ou subversif. D'ailleurs, si les idées et les paroles y circulent sans aucun doute, les autorités saoudiennes s'attachent à contrôler ou réprimer toute manifestation trop ostentatoire du politique.

$\mathrm{Au}$ Moyen-Orient, les mobilités dues aux pèlerinages constituent un champ particulièrement dynamique, dont on n'a présenté ici que quelques pistes à creuser, qui témoignent à la fois de la spécificité des mobilités religieuses, et des transformations globales en cours dans la région. 


\section{BIBLIOGRAPHIE}

ADELKHA Fariba (1997) Iran : vers un espace public confessionnel ? Les Etudes du CERI n²7, juin 1997, 33 p. (littérature grise)

BALCI Bayram (à paraître) « Les communautés ouzbèke et ouighoure d'Arabie Saoudite et leur rôle dans la réouverture des chemins du pèlerinage entre l'Asie centrale et La Mecque ", in Sylvia Chiffoleau et Anna Madoeuf, Les pèlerinages dans le monde arabe, espaces publics, espaces du public?

BERRIANE Mohamed (1992) Tourisme national et migrations de loisir au Maroc (étude géographique), Publication de la Faculté des Lettres et Sciences humaines, Rabat, 498 p.

CLAYER Nathalie et POPOVIC Alexandre (1995) « Le culte d'Ajvatovica et son pèlerinage annuel », in Henri Chambert-Loir et Claude Guillot (dir.), Le culte des saints dans le monde musulman, École française d'Extrême-Orient, pp. 353-365.

GEOFFROY Eric (1995) « Proche-Orient ", in Henri Chambert-Loir et Claude Guillot (dir.), Le culte des saints dans le monde musulman, École française d'Extrême-Orient, pp. 33-56.

HUSSON Laurence (1996) « Les Indonésiens en Arabie Saoudite, pour la foi et le travail », Revue Européenne des Migrations Internationales, vol. 12, n 3, pp. 125-147.

BENNAFLA Karine (à paraître), « L'instrumentalisation du pèlerinage à des fins commerciales : l'exemple du Tchad », in Sylvia Chiffoleau et Anna Madoeuf (dir.), Les pèlerinages dans le monde arabe, espaces publics, espaces du public?

MAYEUR-JAOUEN Catherine (2000) « Tombeau, mosquée et zâwiya : la polarité des lieux saints musulmans ", in André Vauchez (dir.), Lieux sacrés, lieux de culte, sanctuaires. Approches terminologiques, méthodologiques, historiques et monographiques, École française de Rome, pp. 133-147.

\section{AUTEUR}

\section{SYLVIA CHIFFOLEAU}

IFPO/CNRS. IFPO (Institut Français du Proche-Orient, BP344, Damas, Syrie) 